\title{
Light-Front Hamiltonian, Path Integral and BRST Formulations of the Chern-Simons Theory under Appropriate Gauge-Fixing
}

\author{
Usha Kulshreshtha ${ }^{1}$, Daya Shankar Kulshreshtha ${ }^{2}$, James P. Vary ${ }^{3}$ \\ ${ }^{1}$ Department of Physics, Kirori Mal College, University of Delhi, Delhi, India \\ ${ }^{2}$ Department of Physics and Astrophysics, University of Delhi, Delhi, India \\ ${ }^{3}$ Department of Physics and Astronomy, Iowa State University, Iowa, USA \\ E-mail: ushakulsh@gmail.com,dskulsh@gmail.com, jvary@iastate.edu \\ Received August 24, 2010; revised September 17, 2010; accepted October 20, 2010
}

\begin{abstract}
The Chern-Simons theory in two-space one-time dimensions is quantized on the light-front under appropriate gauge-fixing conditions using the Hamiltonian, path integral and BRST formulations.
\end{abstract}

Keywords: Hamiltonian Quantization, Path Integral Quantization, BRST Quantization, Chern-Simons Theories, Light-Cone Quantization, Light-Front Quantization, Constrained Dynamics, Quantum

Electrodynamics Models in Lower Dimensions, Light-Cone Quantization

\section{Introduction}

Studies of the models of quantum electrodynamics in two-space one-time dimensions involving the ChernSimons (CS) theories [1-10] are of wide interest and form a rather broad field of investigations in various contexts. Effective theories with excitations, with fractional statistics are supposed to be described by gauge theories with Chern-Simons term. The statistics (BoseFermi) transmutation has some important experimental consequences in the physics of high $-T_{c}$ superconductivity [3]. Wilczek studied [5] the possibility of exotic statistics appearing in two-space one-time dimensions where the objects obeying this unusual statistics are called anyons [3,5]. The above studies are of very wide interests [1-10] and they provide rather natural motivations for our present studies.

Very recently, we have studied [8-11] the CS theory [8] and the CS-Higgs (CSH) theory in the symmetry phase of the Higgs potential [9] as well as the CSH theory in the so-called broken (or frozen) phase of the Higgs potential [10] using the usual instant-form (IF) of dynamics (on the hyperplanes: $x^{0}=t=$ constant ), under appropriate gauge-fixing conditions.

In the present work we quantize the pure CS theory on the light-front (LF) using the Hamiltonian, path integral and BRST formulations [8-16] under appropriate gaugefixing, using the LF dynamics (on the hyperplanes defined by the LC time: $\left(\tau \equiv x^{+}=\frac{\left(x^{0}+x^{1}\right)}{\sqrt{2}}=\right.$ constant $)$ $[17,18]$. It may be important to mention here that because the LF coordinates are not related to the conventional IF coordinates by a finite Lorentz transformation, the descriptions of the same physical result may be different in the IF and LF dynamics and the LF quantization (LFQ) often has some advantages over the conventional IF quantization (IFQ) and a study of both the IFQ and the LFQ of a theory determines the canonical structure and constrained dynamics of a theory rather completely [8-18].

Different aspects of this theory have been studied by several authors in various contexts [1-10]. For further details of the motivations for a study of the different aspects of the Chern-Simons theories by various authors including a comparative description of different studies, we refer to our earlier work of Reference [8-11]. In the next section, we study its LF Hamiltonian and path integral formulations and its BRST formulation is studied in Section 3. The summary and discussion is finally given in Section 4 . 


\section{Hamiltonian and Path Integral Formulations}

In this section we quantize the pure CS theory on the LF, using the Hamiltonian, path integral formulations under appropriate gauge-fixing. The pure Chern-Simons theory in two-space one-time dimensions is defined by the action [1-10]:

$$
\begin{gathered}
S_{1}=\int \mathcal{L}_{1}\left(A^{\mu}\right) d^{3} x, \mathcal{L}_{1}=\left[\frac{\kappa}{2} \varepsilon^{\mu v \lambda} A_{\mu} \partial_{v} A_{\lambda}\right], \kappa=\left(\frac{\theta}{2 \pi^{2}}\right)(1) \\
g^{\mu \nu}:=\operatorname{diag}(+1,-1,-1) ; \mu, v=0,1,2 ; \varepsilon^{012}=\varepsilon_{012}=+1(2)
\end{gathered}
$$

Here $\theta$ is the Chern-Simons parameter. The LF [5] action of the theory reads:

$$
\begin{aligned}
& S=\int \mathcal{L} d x^{+} d x^{-} d x_{2} \\
& \mathcal{L}:=\left[\frac{\kappa}{2}\left[A^{+} \partial_{+} A_{2}-A^{-} \partial_{-} A_{2}+A^{-} \partial_{2} A^{+}-A^{+} \partial_{2} A^{-}-A_{2} \partial_{+} A^{+}+A_{2} \partial_{-} A^{-}\right]\right]
\end{aligned}
$$

In the following, we would consider the Hamiltonian formulation of the theory described by the above action.
The canonical momenta obtained from the above equation are:

$$
\Pi^{+}:=\frac{\partial \mathcal{L}}{\partial\left(\partial_{+} A^{-}\right)}=0, \Pi^{-}:=\frac{\partial \mathcal{L}}{\partial\left(\partial_{+} A^{+}\right)}=-\frac{\kappa}{2} A_{2}, E\left(\equiv \Pi^{2}\right):=\frac{\partial \mathcal{L}}{\partial\left(\partial_{+} A_{2}\right)}=\frac{\kappa}{2} A^{+}
$$

Here $\Pi^{+}, \Pi^{-}$and $E\left(:=\Pi^{2}\right)$ are the momenta canonically conjugate respectively to $A^{-}, A^{+}$and $A_{2}$.
The above equations however, imply that the theory possesses three primary constraints:

$$
\chi_{1}=\Pi^{+} \approx 0 ; \chi_{2}=\left(\Pi^{-}+\frac{\kappa}{2} A_{2}\right) \approx 0 ; \chi_{3}=\left(E-\frac{\kappa}{2} A^{+}\right) \approx 0
$$

The symbol $\approx$ here denotes a weak equality (WE) in the sense of Dirac [12,13], and it implies that these above constraints hold as strong equalities only on the reduced hypersurface of the constraints and not in the rest of the phase space of the classical theory (and similarly one can consider it as a weak operator equality (WOE) for the corresponding quantum theory).

The canonical Hamiltonian density corresponding to $\mathcal{L}$ is:

$$
\begin{aligned}
\mathcal{H}_{c} & :=\left[\Pi^{+} \partial_{+} A^{-}+\Pi^{-} \partial_{+} A^{+}+E \partial_{+} A_{2}-\mathcal{L}\right] \\
& =\left[\frac{\kappa}{2}\left[A^{+} \partial_{2} A_{-}-A^{-} \partial_{2} A^{+}+A^{-} \partial_{-} A_{2}-A_{2} \partial_{-} A^{-}\right]\right]
\end{aligned}
$$

After including the primary constraints $\chi_{1}, \chi_{2}$ and $\chi_{3}$ in the canonical Hamiltonian density $H_{c}$ with the help of the Lagrange multiplier field $s, u$ and $v$ the total Hamiltonian density $H_{T}$ could be written as:

$$
\begin{aligned}
\mathcal{H}_{T} & =\left[\left(\Pi^{+}\right)_{S}+\left(\Pi^{-}+\frac{\kappa}{2} A_{2}\right) u+\left(E-\frac{\kappa}{2} A^{+}\right) v\right. \\
& \left.+\frac{\kappa}{2}\left[A^{+} \partial_{2} A^{-}-A^{-} \partial_{2} A^{+}+A^{-} \partial_{-} A_{2}-A_{2} \partial_{-} A^{-}\right]\right]
\end{aligned}
$$

The Hamilton's equations of motion of the theory that preserve the constraints of the theory in the course of time could be obtained from the total Hamiltonian (and are omitted here for the sake of bravity):

$$
H_{T}=\int \mathcal{H}_{T} d x^{-} d x_{2}
$$

The preservation of $\chi_{1}, \chi_{2}$ and $\chi_{3}$ for all times does not give rise to any further constraints. The theory is thus seen to possess only three constraints $\chi_{i}$ (with $\mathrm{i}=$ $1,2,3)$, where all $\chi_{i}$ are primary constraints. Further, the matrix of the Poisson brackets among the constraints $\chi_{i}$ is seen to be a singular (in fact, a null) matrix implying that the set of constraints $\chi_{i}$ is first-class and that the theory under consideration is gauge-invariant.

The physical degrees of freedom of the system are governed by the reduced Hamiltonian density of the theory (which is obtained by implementing the constraints of the theory strongly). Also, in the present case, $A^{+}$plays the role of gauge variable and the two pairs $\left(A^{-}, \Pi^{+}\right)$and $\left(A_{2}, E\right)$ are the pair of inessential eliminable variables and a pair describing the physical degrees of freedom of the system. Accordingly, we choose, in the present case, the first pair namely, $\left(A^{+}\right.$, $\left.\Pi^{-}\right)$as the pair describing the physical degrees of freedom and the other pair as the pair of inessential eliminable variables. So for writing the reduced Hamiltonian density of the theory, we choose $A^{-}$and $\Pi^{+}$as the independent variables and the remaining phase space variables as the dependent variables. The later ones are then expressed in terms of the independent variables as: 


$$
\Pi^{+}=0 ; E=\frac{\kappa}{2} A^{+} ; \Pi^{-}=-\frac{\kappa}{2} A_{2}
$$

Finally the reduced Hamiltonian density of the theory describing the physical degrees of freedom of the system expresed in terms of the independent variables is then obtained as:

$$
\mathcal{H}_{R}=\left[2 \Pi^{-} \partial_{-} A^{-}+\kappa A^{+} \partial_{2} A^{-}\right]
$$

where $H_{R}=\int \mathcal{H}_{R} d x$ is the reduced Hamiltonian of the theory and it describes the physical degrees of freedom of the system. Here we remind ourselves that as an alternative to the above, we could have equivalently expressed it in terms of the other pair namely, $\left(A_{2}, E\right)$ instead of the pair $\left(A^{-}, \Pi^{+}\right)$.

Using the above equation we then obtain the field equations derived from the Heisenberg equations of motion as:

$$
\begin{aligned}
& -\partial_{+} \Pi^{+}=i\left[\Pi^{+}, H_{R}\right]=\left(-2 \partial_{-} \Pi^{-}-\kappa \partial_{2} A^{+}\right) \\
& \partial_{+} A^{-}=-i\left[A^{-}, H_{R}\right]=0 \\
& -\partial_{+} \Pi^{-}=i\left[\Pi^{-}, H_{R}\right]=\left(\kappa \partial_{2} A^{-}\right) \\
& \partial_{+} A^{+}=-i\left[A^{+}, H_{R}\right]=\left[2 \partial_{-} A^{-}\right]
\end{aligned}
$$

The vector gauge current of the theory $J^{\mu} \equiv\left(J^{+}, J^{-}, J^{2}\right)$ is:

$$
\begin{aligned}
& J^{+}=\int j^{+} d^{2} x=\int d^{2} x\left[\frac{\kappa}{2}\left(A_{2} \partial_{-} \beta-A^{+} \partial_{2} \beta\right)\right] \\
& J^{-}=\int j^{-} d^{2} x=\int d^{2} x\left[-\frac{\kappa}{2}\left(A_{2} \partial_{+} \beta-A^{-} \partial_{2} \beta\right)\right] \\
& J^{2}=\int j^{2} d^{2} x=\int d^{2} x\left[-\frac{\kappa}{2}\left(A^{-} \partial_{-} \beta-A^{+} \partial_{+} \beta\right)\right]
\end{aligned}
$$

The divergence of the vector gauge current density of the theory could now be easily seen to vanish satisfying the continuity equation: $\partial_{\mu} j^{\mu}=0$, implying that the theory possesses at the classical level, a local vectorgauge symmetry. The action of the theory is indeed seen to be invariant under the local vector gauge transformations:

$$
\begin{aligned}
& \delta A^{-}=-\partial_{+} \beta, \delta A_{2}=-\partial_{2} \beta, \delta s=-\partial_{+} \partial_{+} \beta, \delta u=-\partial_{+} \partial_{-} \beta \\
& \delta A^{+}=-\partial_{-} \beta, \delta \Pi^{+}=o, \delta E=\frac{-\kappa}{2} \partial_{-} \beta, \delta \Pi^{-}=\frac{\kappa}{2} \partial_{2} \beta \\
& \delta v=-\partial_{+} \partial_{2} \beta, \delta \Pi_{s}=\delta \Pi_{u}=\delta \Pi_{v}=0
\end{aligned}
$$

where $\beta \equiv \beta\left(x^{+}, x^{-}, x^{2}\right)$ is an arbitrary function of its arguments. In order to quantize the theory using Dirac's procedure we now convert the set of first-class constraints of the theory $\chi_{i}$ into a set of second-class constraints, by imposing, arbitrarily, some additional constraints on the system called gauge-fixing conditions or the gauge constraints. For this purpose, for the present theory, we could choose, for example, the following set of gauge-fixing condition:

$$
\Psi=A^{-} \approx 0
$$

Here the gauge $A^{-} \approx 0$ represents the light-cone coulomb gauge which is a physically important gauge. Corresponding to this gauge choice, the theory has the following set of constraints under which the quantization of the theory could e.g. be studied:

$$
\begin{aligned}
& \xi_{1}=\chi_{1}=\chi_{1}=\Pi^{+} \approx 0 \\
& \xi_{2}=\chi_{2}=\chi_{2}=\left(\Pi^{-}+\frac{\kappa}{2} A_{2}\right) \approx 0 \\
& \xi_{3}=\chi_{3}=\chi_{3}=\left(E-\frac{\kappa}{2} A^{+}\right) \approx 0 \\
& \xi_{4}=\Psi=A^{-} \approx 0
\end{aligned}
$$

The matrix $R_{\alpha \beta}$ of the Poisson brackets among the set of constraints $\xi_{i}$ with $(i=1,2,3,4)$ is seen to be nonsingular with the determinant given by

$$
\left[\left\|\operatorname{det}\left(R_{\alpha \beta}\right)\right\|\right]^{\frac{1}{2}}=\left[\left[\kappa \delta^{2}\left(x^{-}-y^{-}\right)\right]\left[\delta^{2}\left(x_{2}-y_{2}\right)\right]\right]
$$

The other details of the matrix $R_{\alpha \beta}$ are omitted here for the sake of bravity. Finally, following the standard Dirac quantization procedure, the nonvanishing equal light-cone-time commutators of the theory, under the gauge: $A^{-} \approx 0$ are obtained as:

$$
\begin{aligned}
& {\left[A^{+}\left(x^{+}, x^{-}, x_{2}\right), A_{2}\left(x^{+}, x^{-}, x_{2}\right)\right]} \\
& =\frac{-i}{\kappa} \delta\left(x^{-}-y^{-}\right) \delta\left(x_{2}-y_{2}\right) \\
& {\left[A^{+}\left(x^{+}, x^{-}, x_{2}\right), \Pi^{-}\left(x^{+}, x^{-}, x_{2}\right)\right]} \\
& =\frac{i}{2} \delta\left(x^{-}-y^{-}\right) \delta\left(x_{2}-y_{2}\right) \\
& {\left[\Pi^{-}\left(x^{+}, x^{-}, x_{2}\right), E\left(x^{+}, x^{-}, x_{2}\right)\right]} \\
& =\frac{-i \kappa}{4} \delta\left(x^{-}-y^{-}\right) \delta\left(x_{2}-y_{2}\right)
\end{aligned}
$$

Also, for the later use, for considering the BRST formulation of the theory we convert the total Hamil-tonian density into the first-order Lagrangian density $\mathcal{L}_{I 0}$ :

$$
\begin{aligned}
\mathcal{L}_{I 0} & :=\left[\Pi^{+}\left(\partial_{+} A^{-}\right)+\Pi^{-}\left(\partial_{+} A^{+}\right)+E\left(\partial_{+} A_{2}\right)+\Pi_{s}\left(\partial_{+} s\right)+\Pi_{u}\left(\partial_{+} u\right)+\Pi_{v}\left(\partial_{+} v\right)-H_{T}\right] \\
& =\left[\frac{\kappa}{2}\left[A^{+} \partial_{+} A_{2}-A^{-} \partial_{-} A_{2}+A^{-} \partial_{2} A^{+}-A^{+} \partial_{2} A^{-}-A_{2} \partial_{+} A^{+}+A_{2} \partial_{-} A^{-}\right]\right]
\end{aligned}
$$


In the path integral formulation, the transition to quantum theory is made by writing the vacuum to vacuum transition amplitude for the theory called the generating functional $Z\left[J_{k}\right]$ of the theory [8-11,14,15] under the gauge-fixing under consideration, in the presence of the external sources $J_{k}$ as:

$$
Z\left[J_{k}\right]=\int[d \mu] \exp \left[i \int d^{3} x\left[J_{k} \Phi^{k}+\Pi^{+} \partial_{+} A^{-}+\Pi^{-} \partial_{+} A^{+}+E \partial_{+} A_{2}+\Pi_{s} \partial_{+} s+\Pi_{u} \partial_{+} u+\Pi_{v} \partial_{+} v-H_{T}\right]\right]
$$

Here, the phase space variables of the theory are: $\Phi^{k} \equiv\left(A^{-}, A^{+}, A_{2}, s, u, v\right)$ with the corresponding respective canonical conjugate momenta:
$\Pi_{k} \equiv\left(\Pi^{+}, \Pi^{-}, E, \Pi_{s}, \Pi_{u}, \Pi_{v}\right)$. The functional measure $[d \mu]$ of the generating functional $Z\left[J_{k}\right]$ under the above gauge-fixing is obtained as:

$$
\begin{gathered}
{[d \mu]=\left[\kappa \delta^{2}\left(x^{-}-y^{-}\right) \delta^{2}\left(x_{2}-y_{2}\right)\right]\left[d A^{+}\right]\left[d A^{-}\right]\left[d A_{2}\right][d s][d u][d v]} \\
{\left[d \Pi^{-}\right]\left[d \Pi^{+}\right][d E]\left[d \Pi_{s}\right]\left[d \Pi_{u}\right]\left[d \Pi_{v}\right] \delta\left[\Pi^{+} \approx 0\right]} \\
\delta\left[\left(\Pi^{-}+\frac{\kappa}{2} A_{2}\right) \approx 0\right] \delta\left[\left(E-\frac{\kappa}{2} A^{+}\right) \approx 0\right] \delta\left[A^{-} \approx 0\right]
\end{gathered}
$$

The Hamiltonian and path integral quantization of the theory under the gauge: $A^{-} \approx 0$ is now complete.

\section{BRST Formulation}

For the BRST formulation of the model, we rewrite the theory as a quantum system that possesses the generalized gauge invariance called BRST symmetry. For this, we first enlarge the Hilbert space of our gauge-invariant theory and replace the notion of gauge-transformation, which shifts operators by c-number functions, by a BRST transformation, which mixes operators with Bose and Fermi statistics. We then introduce new anti-commuting variables $\mathrm{c}$ and $\bar{c}$ (Grassman numbers on the classical level and operators in the quantized theory) and a commuting variable $b$ such that $[8-11,16]$ :

$$
\begin{aligned}
& \hat{\delta} A^{-}=-\partial_{+} c, \hat{\delta} A_{2}=-\partial_{2} c, \hat{\delta} v=-\partial_{+} \partial_{2} c, \hat{\delta} s=-\partial_{+} \partial_{+} c \\
& \hat{\delta} A^{+}=-\partial_{-} c, \hat{\delta} \Pi^{+}=o, \hat{\delta} E=-\frac{\kappa}{2} \partial_{-} c, \hat{\delta} \Pi^{-}=\frac{\kappa}{2} \partial_{2} c \\
& \hat{\delta} u=-\partial_{+} \partial_{-} c, \hat{\delta} \Pi_{u}=\hat{\delta} \Pi_{v}=\hat{\delta} \Pi_{s}=0 \\
& \hat{\delta} c=0, \hat{\delta} \bar{c}=b, \hat{\delta} b=0
\end{aligned}
$$

with the property $\hat{\delta}^{2}=0$. We now define a BRSTinvariant function of the dynamical variables to be a function $f$ such that $\hat{\delta} f=0$. Now the BRST gaugefixed quantum Lagrangian density $\mathcal{L}_{B R S T}$ for the theory could be obtained by adding to the first-order Lagrangian density $\mathcal{L}_{T 0}$, a trivial BRST-invariant function, e.g. as follows:

$$
\mathcal{L}_{\text {BRST }}:=\left[\frac{\kappa}{2}\left[A^{+} \partial_{+} A_{2}-A^{-} \partial_{-} A_{2}+A^{-} \partial_{2} A^{+}-A^{+} \partial_{2} A^{-}-A_{2} \partial_{+} A^{+}+A_{2} \partial_{-} A^{-}\right]-\hat{\delta}\left[\bar{c}\left(\partial_{+} A^{-}-\frac{1}{2} b\right)\right]\right]
$$

The last term in the above equation is the extra BRST-invariant gauge-fixing term. After one integra- tion by parts, the above equation could now be written as:

$$
\mathcal{L}_{\text {BRST }}:=\left[\frac{\kappa}{2}\left[A^{+} \partial_{+} A_{2}-A^{-} \partial_{-} A_{2}+A^{-} \partial_{2} A^{+}-A^{+} \partial_{2} A^{-}-A_{2} \partial_{+} A^{+}+A_{2} \partial_{-} A^{-}\right]+\frac{1}{2} b^{2}-b\left(\partial_{+} A^{-}\right)+\left(\partial_{+} \bar{c}\right)\left(\partial_{+} c\right)\right]
$$

Proceeding classically, the Euler-Lagrange equation for $b$ reads:

$$
b=\left(\partial_{+} A^{-}\right)
$$

the requirement $\hat{\delta} b=0$ then implies

$$
\hat{\delta} b=\left[\hat{\delta}\left(\partial_{+} A^{-}\right)\right]
$$

which in turn implies

$$
\partial_{+} \partial_{+} c=0
$$

The above equation is also an Euler-Lagrange equation obtained by the variation of $\mathcal{L}_{\text {BRST }}$ with respect to $\bar{c}$. In introducing momenta one has to be careful in defining those for the fermionic variables. We thus define the bosonic momenta in the usual manner so that

$$
\Pi^{+}:=\frac{\partial}{\partial\left(\partial_{+} A^{-}\right)} \mathcal{L}_{B R S T}=-b
$$

but for the fermionic momenta with directional derivatives we set 


$$
\Pi_{c}:=\mathcal{L}_{\text {BRST }} \frac{\bar{\partial}}{\partial\left(\partial_{+} c\right)}=\partial_{+} \bar{c} ; \Pi_{\bar{c}}:=\frac{\vec{\partial}}{\partial\left(\partial_{+} \bar{c}\right)} \mathcal{L}_{\text {BRST }}=\partial_{+} c
$$

implying that the variable canonically conjugate to $c$ is $\left(\partial_{+} \bar{c}\right)$ and the variable conjugate to $\bar{c}$ is $\left(\partial_{+} c\right)$. For writing the Hamilotonian density from the Lagrangian density in the usual manner we remember that the former has to be Hermitian so that:

$$
\begin{aligned}
H_{B R S T} & =\left[\Pi^{+} \partial_{+} A^{-}+\Pi^{-} \partial_{+} A^{+}+E \partial_{+} A_{2}+\Pi_{s} \partial_{+} s\right. \\
& \left.+\Pi_{u} \partial_{+} u+\Pi_{v} \partial_{+} v+\Pi_{c}\left(\partial_{+} c\right)+\left(\partial_{+} \bar{c}\right) \Pi_{\bar{c}}-L_{B R S T}\right] \\
& =\left[\Pi_{s} \partial_{+} s+\Pi_{u} \partial_{+} u+\Pi_{v} \partial_{+} v-\frac{1}{2}\left(\Pi^{+}\right)^{2}+\Pi_{c} \Pi_{\bar{c}}\right. \\
& \left.+\frac{\kappa}{2}\left[A^{-} \partial_{-} A_{2}-A^{-} \partial_{2} A^{+}+A^{+} \partial_{2} A^{-}-A_{2} \partial_{-} A^{-}\right]\right]
\end{aligned}
$$

The consistency of the last two equations could now be easily checked by looking at the Hamilton's equations for the fermionic variables. Also for the operators $c, \bar{c}, \partial_{+} c$ and $\partial_{+} \bar{c}$, one needs to satisfy the anticommutation relations of $\partial_{+} c$ with $\bar{c}$ or of $\partial_{+} \bar{c}$ with $c$, but not of $c$, with $\bar{c}$. In general, $c$ and $\bar{c}$ are independent canonical variables and one assumes that

$$
\left\{\Pi_{c}, \Pi_{\bar{c}}\right\}=\{\bar{c}, c\}=\partial_{+}\{\bar{c}, c\}=0 ;\left\{\partial_{+} \bar{c}, c\right\}=(-1)\left\{\partial_{+} c, \bar{c}\right\}
$$

where $\{$,$\} means an anticommutator. We thus see that$ the anticommulators in the above equation are non-trivial and need to be fixed. In order to fix these, we demand that c satisfy the Heisenberg equation:

$$
\left[c, \mathcal{H}_{B R S T}\right]=i \partial_{+} c
$$

and using the property $c^{2}=\bar{c}^{2}=0$ one obtains

$$
\left[c, \mathcal{H}_{\text {BRST }}\right]=\left\{\partial_{+} \bar{c}, c\right\} \partial_{+} c
$$

The last three equations then imply :

$$
\left\{\partial_{+} \bar{c}, c\right\}=(-1)\left\{\partial_{+} c, \bar{c}\right\}=i
$$

Here the minus sign in the above equation is nontrivial and implies the existence of states with negative norm in the space of state vectors of the theory. The BRST charge operator $Q$ is the generator of the BRST transformations. It is nilpotent and satisfies $Q^{2}=0$. It mixes operators which satisfy Bose and Fermi statistics. According to its conventional definition, its commutators with Bose operators and its anti-commutators with Fermi operators for the present theory satisfy:

$$
\begin{aligned}
& {\left[A^{+}, Q\right]=\left[A^{-}, Q\right]=\left[A_{2}, Q\right]=\partial_{+} c,} \\
& {\left[\Pi^{-}, Q\right]=-[E, Q]=\frac{\kappa}{2} \partial_{+} c} \\
& \{\bar{c}, Q\}=-\left[\left(\Pi^{+}+\Pi^{-}+E\right)+\frac{\kappa}{2}\left(A_{2}-A^{+}\right)\right]
\end{aligned}
$$

All other commutators and anti-commutators involving $Q$ vanish. In view of this, the BRST charge operator of the present theory can be written as:

$$
Q=\int d x^{-} d x_{2}\left[-i \partial_{+} c\left[\left(\Pi^{+}+\Pi^{-}+E\right)+\frac{\kappa}{2}\left(A_{2}-A^{+}\right)\right]\right]
$$

This equation implies that the set of states satisfying the conditions:

$$
\Pi^{+}|\psi\rangle=0,\left(\Pi^{-}+\frac{\kappa}{2} A_{2}\right)|\psi\rangle=0,\left(E-\frac{\kappa}{2} A^{+}\right)|\psi\rangle=0
$$

belong to the dynamically stable subspace of states $|\psi\rangle$ satisfying $Q \mid \psi>=0$, i.e., it belongs to the set of BRST-invariant states. In order to understand the condition needed for recovering the physical states of the theory we rewrite the operators $c$ and $\bar{c}$ in terms of fermionic annihiliation and creation operators. For this purpose we consider Euler lagrange equation for the variable $c$ derived earlier. The solution of this equation gives (for the light-cone time $x^{+} \equiv \tau$ ) the Heisenberg operators $c(\tau)$ and correspondingly $\bar{c}(\tau)$ in terms of the fermionic Annihilation and creation operators as:

$$
c(\tau)=G(\tau)+F, \bar{c}(\tau)=G^{\dagger} \tau+F
$$

Which at the light-cone time $\tau=0$ imply

$$
\begin{aligned}
& c \equiv c(0)=F, \bar{c}(\tau) \equiv \bar{c}(0)=F^{\dagger} \\
& \partial_{+} c(\tau) \equiv \partial_{+} c(0)=G, \partial_{+} \bar{c}(\tau) \equiv \partial_{+} \bar{c}(0)=G^{\dagger}
\end{aligned}
$$

By imposing the conditions (obtained earlier):

$$
\begin{aligned}
& c^{2}=\bar{c}^{2}=\{\bar{c}, c\}=\left\{\partial_{+} \bar{c}, \partial_{+} c\right\}=0, \\
& \left\{\partial_{+} \bar{c}, c\right\}=(-1)\left\{\partial_{+} c, \bar{c}\right\}=i
\end{aligned}
$$

we then obtain

$$
\begin{gathered}
F^{2}=F^{\dagger 2}=\left\{F^{\dagger}, F\right\}=\left\{G^{\dagger}, G\right\}=0, \\
\left\{G^{\dagger}, F\right\}=(-1)\left\{G, F^{\dagger}\right\}=i
\end{gathered}
$$

Now let $\mid 0>$ denote the fermionic vacuum for which

$$
G|0>=F| 0>=0
$$

Defining $\mid 0>$ to have norm one, the last three equations imply

$$
<0\left|F G^{\dagger}\right| 0>=i, \quad<0\left|G F^{\dagger}\right| 0>=-i
$$

so that

$$
G^{\dagger}\left|0>\neq 0, F^{\dagger}\right| 0>\neq 0
$$

The theory is thus seen to possess negative norm states in the fermionic sector. The existence of these negative norm states as free states of the fermionic part of $\mathcal{H}_{\text {BRST }}$ is , however, irrelevant to the existence of physicsl states in the orthogonal subspace of the Hilbert space. In terms 
of annihilation and creation operators $\mathcal{H}_{\text {BRST }}$ is:

$$
\begin{aligned}
\mathcal{H}_{\text {BRST }} & =\left[\Pi_{s} \partial_{+} s+\Pi_{u} \partial_{+} u+\Pi_{v} \partial_{+} v-\frac{1}{2}\left(\Pi^{+}\right)^{2}+G^{\dagger} G\right. \\
& \left.+\frac{\kappa}{2}\left[A^{-} \partial_{-} A_{2}-A^{-} \partial_{2} A^{+}+A^{+} \partial_{2} A^{-}-A_{2} \partial_{-} A^{-}\right]\right]
\end{aligned}
$$

and the BRST charge operator is:

$$
Q=\int d x^{-} d x_{2}\left[-i G\left[\left(\Pi^{+}+\Pi^{-}+E\right)+\frac{\kappa}{2}\left(A_{2}-A^{+}\right)\right]\right]
$$

Now because $Q \mid \psi>=0$, the set of states annihiliated by $Q$ contains not only the set for which the constraints of the theory hold but also additional states for which

$$
\begin{aligned}
& F|\psi>=G| \psi>=0 \\
& \Pi^{+}|\psi\rangle \neq 0,\left(\Pi^{-}+\frac{\kappa}{2} A_{2}\right)|\psi\rangle \neq 0,\left(E-\frac{\kappa}{2} A^{+}\right)|\psi\rangle \neq 0
\end{aligned}
$$

The Hamiltonian is also invariant under the anti-BRST transformation given by:

$$
\begin{aligned}
& \overline{\hat{\delta}} A^{-}=\partial_{+} \bar{c}, \overline{\hat{\delta}} A_{2}=\partial_{2} \bar{c}, \overline{\hat{\delta}} s=\partial_{+} \partial_{+} \bar{c}, \overline{\hat{\delta}} v=\partial_{+} \partial_{2} \bar{c} \\
& \overline{\hat{\delta}} A^{+}=\partial_{-} \bar{c}, \overline{\hat{\delta}} \Pi^{+}=0, \overline{\hat{\delta}} \Pi^{-}=-\frac{\kappa}{2} \partial_{2} \bar{c}, \overline{\hat{\delta}} E=\frac{\kappa}{2} \partial_{-} \bar{c} \\
& \overline{\hat{\delta}} u=\partial_{+} \partial_{-} \bar{c}, \overline{\hat{\delta}} \Pi_{s}=\overline{\hat{\delta}} \Pi_{u}=\overline{\hat{\delta}} \Pi_{v}=0 \\
& \overline{\hat{\delta}} \bar{c}=0, \overline{\hat{\delta}} c=-b, \overline{\hat{\delta}} b=0
\end{aligned}
$$

with generator or anti-BRST charge

$$
\bar{Q}=\int d x^{-} d x_{2}\left[i \partial_{+} \bar{c}\left[\left(\Pi^{+}+\Pi^{-}+E\right)+\frac{\kappa}{2}\left(A_{2}-A^{+}\right)\right]\right]
$$

which in terms of annihilation and creation operators reads:

$$
\bar{Q}=\int d x^{-} d x_{2}\left[i G^{\dagger}\left[\left(\Pi^{+}+\Pi^{-}+E\right)+\frac{\kappa}{2}\left(A_{2}-A^{+}\right)\right]\right]
$$

We also have

$$
\partial_{+} Q=\left[Q, H_{B R S T}\right]=0 ; \partial_{+} \bar{Q}=\left[\bar{Q}, H_{B R S T}\right]=0
$$

with

$$
H_{\text {BRST }}=\int d x^{-} d x_{2} \mathcal{H}_{\text {BRST }}
$$

and we further impose the dual condition that both $Q$ and $\bar{Q}$ annihilate physical states, implying that:

$$
Q \mid \psi>=0 \text { and } \bar{Q} \mid \psi>=0
$$

The states for which the constraints of the theory hold, satisfy both of these conditions and are in fact, the only states satisfying both of these conditions, since although with

$$
G^{\dagger} G=(-1) G G^{\dagger}
$$

there are no states of this operator with $G^{\dagger} \mid \psi>=0$ and $F^{\dagger} \mid \psi>=0$, and hence no free eigenstates of the fermionic part of $\mathcal{H}_{B R S T}$ that are annihiliated by each of $G, G^{\dagger}, F$, and $F^{\dagger}$. Thus the only states satisfying $Q \mid \psi>=0$ and $\bar{Q} \mid \psi>=0$ are those that satisfy the constraints of the theory. Now because $Q|\psi\rangle=0$, the set of states annihilated by $Q$ contains not only the set of states for which the constraints of the theory hold but also additional states for which the constraints of the theory do not hold in particular. This situation is, however, easily avoided by aditionally imposing on the theory, the dual condition: $Q \mid \psi>=0$ and $\bar{Q} \mid \psi>=0$. Thus by imposing both of these conditions on the theory simultaneously, one finds that the states for which the constraints of the theory hold satisfy both of these conditions and, in fact, these are the only states satisfying both of these conditions because in view of the conditions on the fermionic variables $c$ and $\bar{c}$ one cannot have simultaneously $c, \partial_{+} c$ and $\bar{c}, \partial_{+} \bar{c}$, applied to $|\psi\rangle$ to give zero. Thus the only states satisfying $Q \mid \psi>=0$ and $\bar{Q} \mid \psi>=0$ are those that satisfy the constraints of the theory and they belong to the set of BRST-invariant as well as to the set of anti-BRSTinvariant states.

Alternatively, one can understand the above point in terms of fermionic annihiliation and creation operators as follows. The condition $Q \mid \psi>=0$ implies that the set of states annihiliated by $Q$ contains not only the states for which the constraints of the theory hold but also additional states for which the constraints do not hold. However, $\bar{Q} \mid \psi>=0$ guarantees that the set of states annihiliated by $\bar{Q}$ contains only the states for which the constraints hold, simply because $G^{\dagger} \mid \psi>\neq 0$ and $F^{\dagger} \mid \psi>\neq 0$. Thus in this alternative way also we see that the states satisfying $Q|\psi\rangle=\bar{Q}|\psi\rangle=0$ are only those states that satisf the constraints of the theory and also that these states belong to the set of BRST- invariant and anti-BRST invariant states. This completes the BRST formulation of the theory.

\section{Summary and Discussion}

IFQ of the present theory has been studied by us in Reference [8] (on the hyperplanes $x^{0}=t=$ constant $[17,18]$ ). In the present work the theory has been quantized using the LF dynamics (on the the hyperplanes of the LF defined by the light-cone time $x^{+} \equiv \tau=\left(x^{0}+x^{1}\right) / \sqrt{2}=$ constant $[17,18]$. It is important to mention here that a study of both of the IFQ and LFQ for a theory really determines the dynamics of the system (a la Dirac) completely, necessitating the present study. For further details on the Dirac's different rela- tivistic forms of dynamics, we refer to the work of Reference $[17,18]$. 
The LFQ has several advantages over the convensional IFQ. In particular, for a LF theory seven out of ten Poincare generators are kinematical while the IF theory has only six kinematical generators $[17,18]$. In our treatment, we have made the convention to regard the lightcone variable $x^{+} \equiv \tau$ as the LF time coordinate and the light-cone variable $x^{-}$has been treated as the longitudinal spatial coordinate. The temporal evolution of the system in $x^{+}$is generated by the total Hamil- tonian of the system.

The constrained dynamics of our LF theory reveals that it possesses a set of three constraints which are primary. Also there is no secondary Gauss law constraint in the theory. The matrix of the Poission brackets of these three constraints is singular implying that they form a set of first-class constraints. This implies in turn, that the corresponding theory is gauge-invariant. The theory is indeed seen to possess a local vector gauge symmetry, and correspondingly there exists a conserved local vector gauge current.

Now because the set of constraints of the theory is first-class, one could quantize the theory under some suitable gauge-fixing as we have done in our present work for the Hamiltonian and path integral quantization of our theory. For this we have choosen the gauge $A^{-} \approx 0$. The gauge $A^{-} \approx 0$ represents the light-cone coulomb gauge. This gauge choice is not only acceptable and consistent with our quantization procedures but is also a physically more intersting gauge choice representing the light-cone coloumb gauge.

However, in the above Hamiltonian and path integral quantization of the theory under some gauge-fixing conditions the gauge-invariance of the theory gets broken because the procedure of gauge-fixing converts the set of first-class constraints of the theory into a set of secondclass one, by changing the matrix of the Poission brackets of the constraints of the theory from a singular one into a non-singular one. In view of this, in order to achieve the quantization of our gauge-invariant theory, such that the gauge-invariance of the theory is maintained even under gauge-fixing, one of the possible ways is go to a more generalized procedure called the BRST quantization [8-11,16], where the extended gauge symmetry called as the BRST symmetry is maintained even under gauge-fixing. It is therefore desirable to achieve this so-called BRST quantization also if possible. This therefore makes a kind of complete quantization of a theory. The light-cone BRST quantization of the present theory has been studied by us in the present work, under some specfic gauge choice (where a particular gauge has been choosen by us and which is not unique by any means). In this procedure, when we embed the original gauge-invariant theory into a BRST system, the quantum
Hamiltonian density $\mathcal{H}_{\text {BRST }}$ (which includes the gaugefixing contribution) commutes with the BRST charge as well as with the anti-BRST charge. The new (extended) gauge symmetry which replaces the gauge invariance is maintained (even underthe BRST gauge-fixing) and hence projecting any state onto the sector of BRST and anti-BRST invariant states yields a theory which is isomorphic to the original gauge-invariant theory.

In conclusion, in the present work we have constructed the quantum theory corresponding to the classical ChernSimons theory defined by the action (1) (or equivalently defined by the LF action (2)) by quantizing the corresponding classical theory using three different quantization procedures called the Hamiltonian, path integral and BRST formulations using the LF quantization on the hyperplanes of the LF defined by the LC-time $x^{+}=\tau=$ constant . In the LF Hamiltonian quantization of the theory we have obtained the nonvanishing equal LC time commutators (given by the Equation (16)) of the LF theory (defined by Equation (2)). In the Path integral quantization of the theory we have explicitly constructed the vacuum to vacuum transition amplitude of the theory called the generating functional of the theory given by Equations (18) and (19). In the BRST quantization, we have explicitly constructed the BRST gauge-fixed quantum Lagrangian of the theory given by Equation (21) (or equivalently by Equation (22)). The quantum BRST-Hamiltonian of the theory has also been constructed given by Equations (28) (or equivalently by Equation (43)). The BRST and anti-BRST charge operators of the theory have also been constructed defined respectively by Equation (34) (or equivalently by Equation (44)) and Equation (47) (or equivalently by Equation (48)). The methods of IFQ and LFQ are pioneered by non other than Dirac [17,18], where the advantages of LFQ over the IFQ have also been discussed. The reasons for the LFQ versus the usual IFQ are best explained in the rather recent review by Brodsky et al. [18] as well as in our earlier work $[11,14,15]$. The physical applications of these studies of the CS theory in various contexts have already been discussed in the introduction. The above points illustrate very clearly the reasons and motivations for the present studies.

\section{References}

[1] G. V. Dunne, “Aspects of Chern-Simons Theories," hep-th/9902115, and references therein.

[2] A. Smirnov, "Notes on Chern-Simons Theory in the Temporal Gauge”, arXiv: hep-th/09105011.

[3] E. J. Ferrer, R. Hurka and V. D. L. Incera, "High Temperature Anyon Superconductivity," Modern Physics Letters B, Vol. 11, No. 1, 1997, pp. 1-8. 
[4] R. B. Laughlin, "Nobel Lecture: Fractional Quantization," Reviews of Modern Physics, Vol. 71, No. 4, 1999, pp. 863-874.

[5] F. Wilczek, "Quantum Mechanics of Fractional Spin Particles,” Physical Review Letters, Vol. 49, No. 14, 1982, pp. 957-959.

[6] D. Boyanovsky, "Chern-Simons with Matter Fields", Phyical Review, Vol. 42, No. 4, 1990, pp. 1179-1183.

[7] D. Boyanovsky, E. T. Newman and C. Rovelli, "On the Quantization of Dynamical Systems with Chern-Simons Terms,” Physical Review, D45, 1992, pp. 1210-1216.

[8] U. Kulshreshtha and D. S. Kulshreshtha, "Hamiltonian, Path Integral and BRST Formulations of the Chern-Simons Theory under Appropriate Gauge-Fixing,” Canadian Journal of Physics, Vol. 86, No. 2, 2008, pp. 401-407.

[9] U. Kulshreshtha, D. S. Kulshreshtha, H. J. W. Mueller-Kirsten and J. P. Vary, "Hamiltonian, Path Integral and BRST Formulations of the Chern-Simons Higgs Theory under Appropriate Gauge-Fixing,” Physica Scripta, Vol. 79, No. 4, 2009.

[10] U. Kulshreshtha, "Hamiltonian, Path Integral and BRST Formulations of the Chern-Simons Higgs Theory in the Broken Symmetry Phase," Physica Scripta, Vol. 75, No. 6, 2007, pp. 795-802.

[11] U. Kulshreshtha, D. S. Kulshreshtha and J. P. Vary, "Light-Front Hamiltonian, Path Integral and BRST Formulations of the Chern-Simons Higgs Theory under Ap- propriate Gauge-Fixing,” Physica Scripta, 82:055101, 2010.

[12] P. A. M. Dirac, "Generalized Hamiltonian Dynamics," Canadian Journal of Mathematics, Vol. 2, 1950, pp. 129-148.

[13] M. Henneaux and C. Teitelboim, "Quantization of Gauge Systems,” Princeton University Press, New Jersey, 1992.

[14] U. Kulshreshtha and D. S. Kulshreshtha, "Conformally Gauge-Fixed Polyakov D1 Brane Action in the Presence of a 2-Form Gauge Field: The Instant-Form and FrontForm Hamiltonian and Path Integral Formulations," Physics Letters B, Vol. 555, No. 3-4, 2003, pp. 255-263.

[15] U. kulshreshtha and D. S. Kulshreshtha, "Hamiltonian and Path Integral Formulations of the Dirac-Born-Infeld Nambu-Goto D1 Brane Action with and without a Dilaton Field under Gauge-Fixing,” The European Physical Journal C, Vol. 29, No. 3, 2003, pp. 453-461.

[16] D. Nemeschansky, C. Preitschopf and M. Weinstein, “A BRST Primer,” Annals of Physics, Vol. 183, No. 2, 1988, pp. 226-268.

[17] P. A. M. Dirac, "Forms of Relativistic Dynamics," Reviews of Modern Physics, Vol. 21, No. 3, 1949, pp. 392-399.

[18] S. J. Brodsky, H. C. Pauli and S. S. Pinsky, "Quantum Chromodynamics and other Field Theories on the Light-Cone”, Physics Reports, Vol. 301, No. 4-6, 1998, pp. 299-486. 